\title{
Insight of the Knot of Henry: A Case Report
}

\author{
Shalini Kumar*, Adil Asghar, Farah Kausar, Ashar Imam, Khalid Mehmood \\ and Ram Prakash \\ Department of Anatomy, Hamdard Institute of Medical Sciences and Research (HIMSR),NewDelhi,India \\ *Correspondence:Dr.Shalini Kumar,Assistant Professor,Department of Anatomy,HIMSR,Jamia Hamdard,New \\ Delhi-110062,India,
}

\begin{abstract}
Tendon transfer of flexor digitorum longus tendon(FDLT) or flexor hallucis longus tendon(FHLT) into the tibialis posterior tendon is carried out in patients with tibialis posterior dysfunction. FDLT and FHLT are connected in the region of the Knot of Henry. The present study has investigated the anatomical variations of this tendinous interconnection. The results could be used to determine which of two tendons should be transected proximal to the region of knot of henry in surgical treatment of tibialis posterior dysfunction. The transaction of FDLT /FHLT proximal to region of Knot of Henry for repair of tibialis posterior dysfunction would result in retention of function of the hallux and lesser digits in majority of cases.

Key words:FDLT- flexor digitorum longus tendon,FHLT-flexor hallucis longus tendon,Knot of henry
\end{abstract}

\section{Background}

A complex variable interconnection between the long flexors of toes occurs in the midfoot. The region of cross connection between the flexor digitorum longus tendon(FDLT) and flexor hallucis longus tendon(FHLT) in the midfoot has been termed the " master knot " (Henry 1995) ${ }^{1}$. The dynamics of digit movement in different anatomical variations at the region of knot of Henry may affect the outcome of surgical intervention in this area. Tibialis posterior dysfunction is probably the most common cause of acquired flatfoot in adults, possibly aggravated by the unopposed exerting force of peroneus brevis (Coughling and Mann, 1999) $)^{2}$. During high loads, such as take off phase of walking Tibialis posterior forms a dynamic reserve to ligamentous support of the longitudinal arch(Basmajian and Stecko 1963). Many early cases of acquired flat foot in adults can be managed conservatively with biomechanical realignment using a functional foot orthosis. If this fails, or in more advanced disease, the main surgical options are synovectomy, tendon transfer and arthrodesis of the subtalar joint with or without talonavicular and calcaneocuboid joint fusion(triple arthrodesis). Tendon transfer often forms part of the operative treatment of planovalgus hindfoot collapse that still has correctability movement at subtalar joint. Tendon transfer of FDLT or FHLT into the tibialis posterior tendon is accepted practice for the treatment of Tibialis posterior dysfunction, but there is some controversy over which is best (Goldner 1985, Mann and Thompson,1985) (,5 $^{4}$. This study is unique because it relates the types of anatomical variation in the region of Knot of Henry to the digital plantar flexion movements, which may have implication for the way acquired flatfoot is treated surgically. A test involving traction applied to the proximal parts of FDLT and FHLT at the time of surgery may provide a basis for selection of either flexor digitorum longus or flexor hallucis longus tendon as a muscle/ tendon unit for transfer.

\section{Dissection}

Skin, superficial fascia and plantar aponeurosis were removed from embalmed male cadaver. The tendons of Flexor digitoum longus and Flexor hallucis longus were dissected. The region of the intersection of two tendons in the region of Knot of Henry was carefully dissected. This area was photographed using a digital camera.

Results: On examination of tendons in region of Knot of Henry showed a slip arising from FHLT to FDLT(fig.1\&2). In left foot the slip reaches the distal phalynx of the second toe(fig.2\&3).

\section{Discussion}

Knowledge of the tendinous variations in the region of the Knot is probably important in the tendon transfer method used in the surgical treatment of tibialis posterior insufficiency. The FDLT is accessible as it is close anatomically to the tibialis posterior tendon but has the disadvantage that FDL has only half the strength of FHLT and a quarter of the strength of tibialis posterior. Richardson $(1998)^{6}$ reported good results in surgical treatment of pes planus by anterior calcaneal osteotomy and tendon transfer of either FHLT or FDLT.

Our result shows that if tendon transfer is chosen for surgical treatment of tibialis posterior dysfunction, transaction of FDLT would probably still leave the hallux functional, the same cannot be said for sacrifice of FHLT. This study did not set out to advise on surgical techniques for reconstituting the tibialis 
posterior, but if an intraoperative test of pulling on each tendon in turn indicates that there will be a deficit in toe flexion control after the preferred tendon has been divided consideration could be given to suturing the distal stump to the remaining intact tendon. For example if one's preference is to transect the FHLT but the intraoperative assessments of FHLT and FDLT indicate that the FHLT has predominant function, one option would be to suture the distal stump of FHLT(after transection) to the intact FDLT, thus preserving hallux function. A tenodesis is often carried out proximal to the region of the Knot of Henry before sectioning the chosen tendon (Cobb, 1996) ${ }^{7}$. Assuming that such a tenodesis is successful, and then the clinical question related to functional deficit is of less interest.

In conclusion, an awareness of the existence of variations in the region of the Knot of Henry is necessary for practicing foot surgeons. We would suggest that if a decision has been made to use tendons of either FHLT or FDLT for tibialis posterior reconstruction, the use of either FHLT or FDLT should not be based on dogma but rather on assessment of tendon predominance at the time of surgery and functional needs of the patient.

\section{References}

[1]. $\quad$ Henry AK. 1995 Extensile exposure. $3^{\text {rd }}$ Ed. Edinburgh: Churchill Livingstone. p. 300-308.

[2]. Coughlin MJ, Mann RA 1999. Surgery of the foot and ankle, $7^{\text {th }}$ Ed. Vol. 1. St. Louis: Mosby. p. 745-758.

[3]. Basmajian VJ, Stecko G, 1963. The role of the muscle in arch support of the foot: an electromyographic study. J Bone Joint Surg 45A: $1184-1190$

[4]. Goldner JL. 1985. Letter to the editor. J Bone Joint Surg 67A: 1448

[5]. Mann RA, Thompson FM. 1985. Rupture of the posterior tibial tendon causing flat foot, J Bone Joint Surg 67 A: 556-561.

[6]. Richardson EG. 1998. Disorders of the hallux. In: Canale ST, editor. Campbell's operative orthopaedics. $9^{\text {th }}$ Ed. St. Louis: Mosby Year Book. P 1712-1745.

[7]. Cobb N. 1996. Tibialis posterior tendon disorders. In Helal B. editor. Surgery of disorders of the foot and ankle. London: Lippincott- Raven. P 291-302.
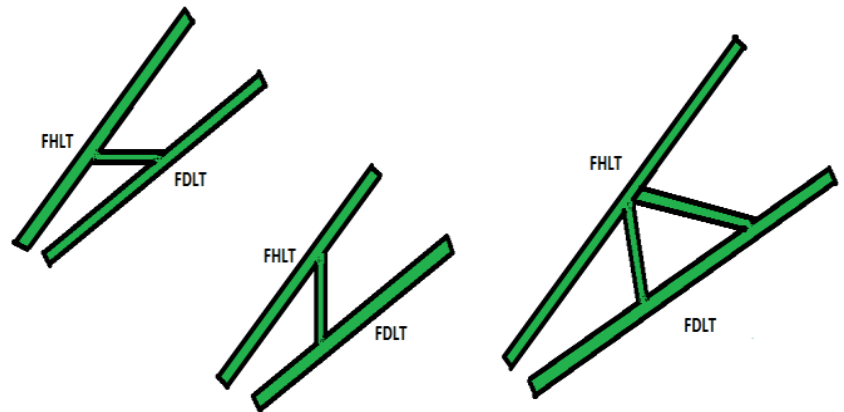

Figure 1: Diagrams to illustrate the direction of the tendinous slips between Flexor hallucis longus tendon (FHLT) and Flexor digitorum longus tendon (FDLT).
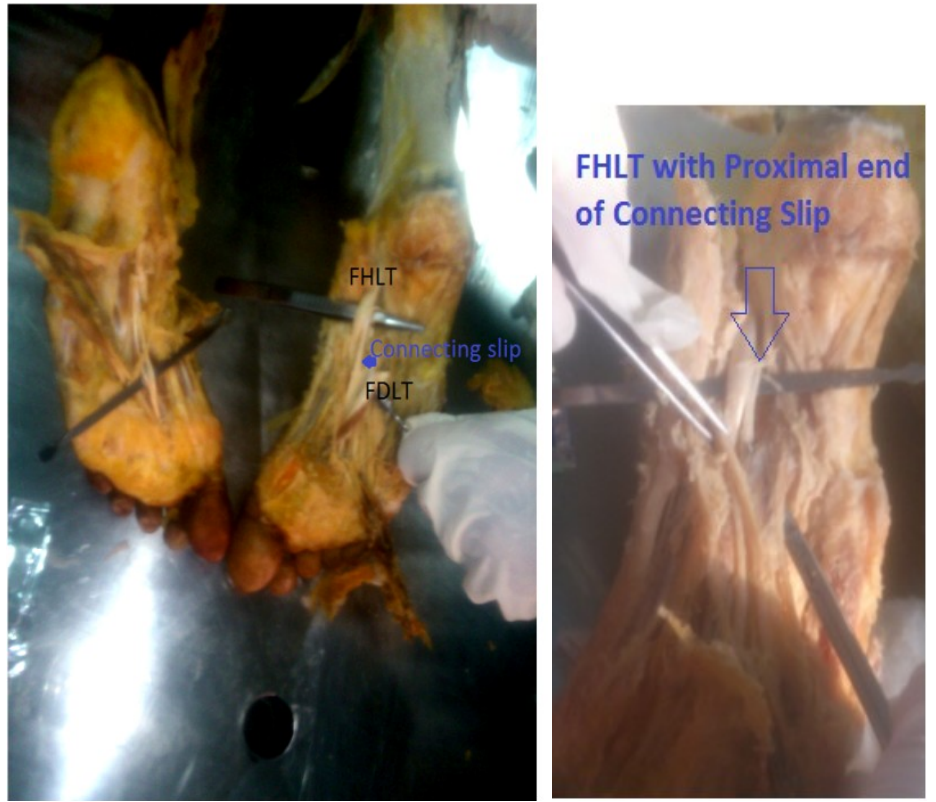

Fig2. A connecting slip from FHLT to FDLT and FHLT with proximal end of connecting slip 


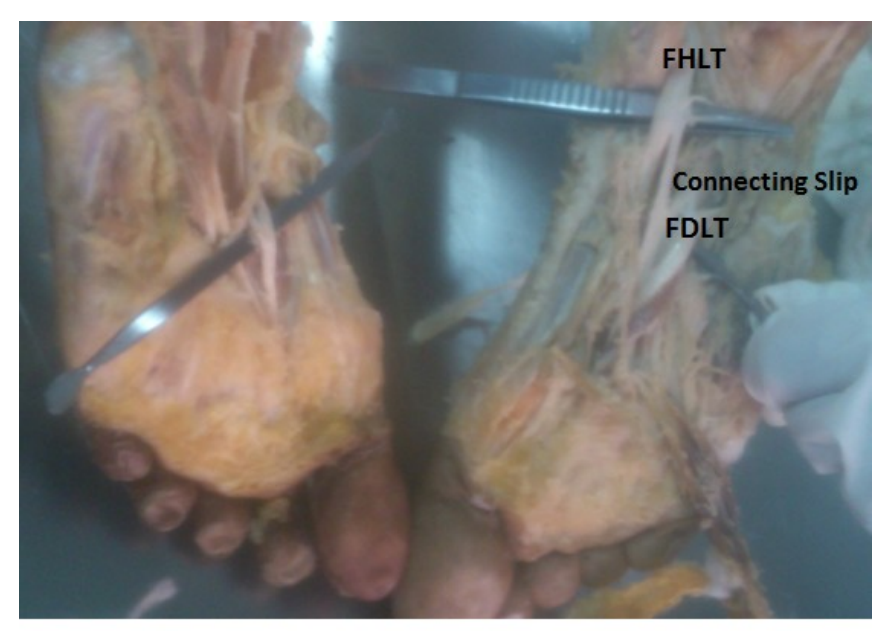

Fig:3 a connecting slip from FHLT to FDLT . 